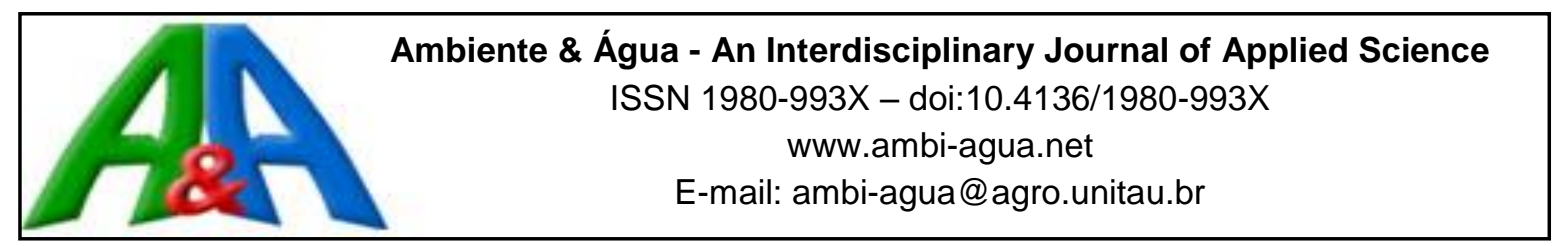

\title{
Análise de BTEX em água: comparação entre duas colunas cromatográficas
}

\author{
doi: 10.4136/ambi-agua.1171
}

Received: 16 Aug. 2013; Accepted: 24 Feb. 2014

\section{Danielle de Almeida Carvalho ${ }^{1 *}$; Rosália Maria de Oliveira ${ }^{1}$; Célia Regina Sousa da Silva ${ }^{2}$; Priscila Tamiasso Martinhon²; Sérgio Alves da Silva ${ }^{1}$}

\footnotetext{
${ }^{1}$ Escola Nacional de Saúde Pública Sergio Arouca, FIOCRUZ - Rio de Janeiro, RJ, Brasil

${ }^{2}$ Universidade Federal do Rio de Janeiro - Rio de Janeiro, RJ, Brasil.

*Autor correspondente: e-mail: danny@ensp.fiocruz.br,

rosalia@ensp.fiocruz.br, sousa@iq.ufrj.br, pris-martinhon@ hotmail.com, sergio.silva@incqs.fiocruz.br
}

\section{RESUMO}

Este estudo teve como objetivo a implementação e a validação de uma metodologia por CG-DIC para a determinação de Benzeno, Tolueno, Etilbenzeno e Xilenos, BTEX, em água potável, como indicadores de contaminação. A metodologia utilizada foi realizada em quatro etapas: a primeira consistiu na separação adequada dos analitos da matriz comparando duas colunas cromatográficas com características diferentes (Supelcowax ${ }^{\mathrm{TM}} 10$ e HP-5); a segunda, na extração dos seis compostos (BTEX) variando o tipo de solvente utilizado, o tempo e a velocidade de extração; as duas últimas etapas consistiram, respectivamente, no tratamento estatístico dos dados obtidos e na validação da metodologia. A coluna Supelcowax ${ }^{\mathrm{TM}} 10$ apresentou melhores resultados na separação dos BTEX. A recuperação mais eficiente foi obtida usando microextração líquido-líquido utilizando o isooctano como solvente, cujo percentual variou de 57,8 a $89,8 \%$. A linearidade foi observada para todos os compostos estudados na faixa de trabalho.

Palavras-chave: contaminação da água potável, microextração líquido-líquido.

\section{Analysis of BTEX in water: comparison between two chromatographic columns}

\section{ABSTRACT}

This study aimed to implement and validate the GC-FID methodology to determine benzene, toluene, ethylbenzene and xylenes, or "BTEX," as contamination indicators in drinking water. The methodology included four steps: the first was to adequately separate analytes from the matrix by comparing two different chromatography columns (Supelcowax $^{\mathrm{TM}} 10$ e HP-5); the second step was the extraction of six compounds of (BTEX) using various types of solvents, extraction times and speeds; the third step statistically analyzed the resulting data; and the final step validated the methodology. Column Supelcowax ${ }^{\mathrm{TM}} 10$ had better results in BTEX separation. The most effective recovery was achieved by using liquid-liquid micro-extraction with isooctane as solvent, which resulted in a 
recovery percentage of 57.8 to $89.8 \%$. Linearity was observed for all compounds studied in the work range.

Keywords: drinking water contamination, liquid-liquid micro-extraction.

\section{INTRODUÇÃO}

Com o crescente aumento da população e da atividade industrial, o tratamento e condicionamento da água potável, assim como a proteção do meio ambiente, é uma das principais preocupações da sociedade moderna. O fornecimento adequado de água potável é uma das necessidades primárias para uma boa saúde e, nesse contexto, a água subterrânea é uma fonte alternativa de abastecimento de água para o consumo humano (Gobato e Lanças, 2001). Em geral, as águas subterrâneas são potáveis e dispensam tratamento prévio; no entanto, quando sua exploração é realizada em área urbana, os maiores problemas de contaminação destes mananciais são atribuídos ao vazamento de combustíveis dos tanques de armazenamento subterrâneo dos postos de gasolina (Silva et al., 2002).

A contaminação da água subterrânea por gasolina é atribuída aos hidrocarbonetos aromáticos, que são os compostos mais solúveis e móveis da fração da gasolina, tais como a mistura BTEX, de conhecido potencial de contaminação e toxicidade à saúde humana e ambiental. Os efeitos adversos da exposição humana a essas substâncias incluem danos ao fígado, rins, coração, pulmões e sistema nervoso (WHO, 1996a; 1996b; 2000a; 2000b; 2004).

No Brasil, devido ao grande aumento do número de postos de combustíveis surgidos nas décadas de 70 e 80 , supõe-se que a vida útil dos tanques de armazenamento esteja próxima do final, aumentando a possibilidade de vazamentos (Lourenço, 2005), mesmo porque, estes contaminantes são os que primeiro atingem o lençol freático (Corseuil e Marins, 1997).

Assim, as responsabilidades relativas ao controle e vigilância da qualidade da água que chega às residências é obrigação legal das empresas de abastecimento e segue a Norma de Potabilidade regulamentada pela Portaria $\mathrm{N}^{\circ}$ 2914, de 12 de dezembro de 2011, do Ministério da Saúde. Segundo a Lei No 11.097, de 13/01/2005, publicada no Diário Oficial da União (DOU), em 14/01/2005, é missão da Agência Nacional de Petróleo a regulação, a contratação e a fiscalização das atividades econômicas das indústrias do petróleo, do gás natural e dos biocombustíveis, inclusive a promoção de estudos visando à delimitação de espaços para efeito de concessão das atividades de exploração, desenvolvimento e produção, de modo a preservar o interesse nacional, garantir o abastecimento e proteger os consumidores quanto ao preço e a qualidade dos produtos.

A Tabela 1 mostra a Concentração Máxima Permitida dos BTEX em água para consumo humano em diferentes países.

Tabela 1. Concentração Máxima Permitida dos BTEX em água para consumo humano em diferentes países e documentos orientadores em $\mu \mathrm{g} \mathrm{L}^{-1}$.

\begin{tabular}{l|cccccc}
\hline \multirow{2}{*}{ Substância } & USEPA $^{1}$ & $\begin{array}{c}\text { CONAMA } \\
357 / 2005^{2}\end{array}$ & $\begin{array}{c}\text { Portaria } \\
2914 / 2011^{3}\end{array}$ & $\begin{array}{c}\text { Directive } \\
98 / 83 / \mathrm{EC}^{4}\end{array}$ & ADWG $^{5}$ & GCDWQ $^{6}$ \\
\cline { 2 - 7 } & EstadosUnidos & Brasil & Brasil & $\begin{array}{c}\text { Comunidade } \\
\text { Européia }\end{array}$ & Austrália & Canadá \\
\hline Benzeno & 5 & 5 & 5 & 1 & 1 & 5 \\
Tolueno & 1000 & 2 & 170 & - & 800 & - \\
Etilbenzeno & 700 & 90 & 200 & - & 300 & - \\
Xilenos & 10.000 & 300 & 300 & - & 600 & - \\
\hline
\end{tabular}

Fontes: ${ }^{1}$ USEPA (2004); ${ }^{2}$ Brasil (2005); ${ }^{3}$ Brasil (2011b); ${ }^{4}$ European Communities (1998); ${ }^{5}$ Australia (2004); ${ }^{6}$ Canada (1996). 
A preocupação principal após um vazamento de combustível é a de tomar providências para circunscrevê-lo à menor área possível, o que facilitará os trabalhos de remediação e, em caso de fonte receptora de água subterrânea, poderá evitar a utilização de água contaminada; tais iniciativas têm o objetivo de preservar a saúde coletiva, minimizando os impactos ambientais e os custos de recuperação da área atingida (Silva, 2002).

Existem várias técnicas desenvolvidas para análise dos compostos BTEX em água. Porém, a maioria depende de equipamentos caros e sofisticados como purge and trap e headspace para a sua detecção. A proposta deste trabalho visa o desenvolvimento e a validação de uma técnica simples de identificação e de quantificação desses compostos, de forma mais rápida e barata do que as já citadas, o que poderá viabilizar a análise em laboratórios dos órgãos reguladores do Brasil, bem como facilitará o controle dos processos de remediação e avaliação da eficiência do mesmo.

A necessidade de métodos de ensaio validados é clara, embora os mecanismos para o desenvolvimento de uma validação intralaboratorial adequada não estejam bem acordados internacionalmente. A maior parte das publicações que tratam de validações intralaboratoriais de métodos de ensaio limitam-se às definições dos principais parâmetros de desempenho e a roteiros genéricos de procedimentos de validação (Green, 1996; EURACHEM, 1998).

A Norma NBR ISO/IEC 17.025 estabelece que devam ser validados métodos utilizados fora dos escopos para os quais foram concebidos e que tenham sido ampliados ou modificados; a validação também se faz necessária em métodos ainda não normatizados e em métodos criados ou desenvolvidos em laboratórios. Entretanto, a velocidade nos avanços científicos e tecnológicos, associada à crescente necessidade de garantia da qualidade gera dificuldades para que os estudos colaborativos acompanhem as demandas, tanto por questões técnicas e organizacionais, quanto por questões infra-estruturais e financeiras.

O presente estudo tem como objetivo o desenvolvimento de uma nova metodologia de extração para a determinação de Benzeno, Tolueno, Etilbenzeno e os isômeros orto, meta e para do Xileno, comumente denominados de BTEX, em água para consumo humano, em níveis de concentração exigidos pela Norma de Potabilidade vigente. Utilizou-se a técnica de Cromatografia à Gás de Alta Resolução por Detecção de Ionização por Chama (CGAR-DIC) como metodologia de análise que foi adequada, implementada e validada. O processo de validação utilizado foi intralaboratorial, tendo como base o documento orientativo do Instituto Nacional de Metrologia e da Eurachem Working Group, além da NBR ISO/IEC 17.025 e estudos publicados na literatura científica.

\section{MATERIAIS E MÉTODOS}

\subsection{Equipamentos e Acessórios}

Cromatógrafo à Gás de Alta Resolução HP 6890, equipado com sistema de injeção automática e Detector de Ionização por Chama; Balança analítica digital - Mettler Toledo AG245, calibrada antes das pesagens com pesos padrões, certificados pelo INMETRO. Placa de aquecimento e agitação Thermolyne NUOVA Stir Plate.

\subsection{Reagentes e Substâncias de Referência utilizados}

Acetona grau de pureza para análise de resíduos; Sulfato de sódio anidro granulado grau de pureza para análise de resíduos (previamante seco em mufla a $400{ }^{\circ} \mathrm{C}$ por $12 \mathrm{~h}$ ); Isooctano grau de pureza para análise de traços orgânicos; Hexano grau de pureza para análise de traços orgânicos; 1-Heptanol grau pureza 98\%; 1-Octanol grau pureza para análise; Coluna Supelcowax $^{\mathrm{TM}} 10$, com $60 \mathrm{~m}$ de comprimento, 0,32 $\mathrm{mm}$ de diâmetro interno e 0,50 $\mu \mathrm{m}$ de espessura de filme; Coluna HP-5 com $30 \mathrm{~m}$ de comprimento, $0,32 \mathrm{~mm}$ de diâmetro interno e $0,25 \mu \mathrm{m}$ de espessura de filme. 


\subsection{Metodologia}

A metodologia utilizada foi realizada em 4 etapas: a primeira etapa consistiu na separação adequada dos analitos da matriz comparando duas colunas cromatográficas com características diferentes - Supelcowax ${ }^{\mathrm{TM}} 10$ e HP-5; a segunda etapa consistiu da extração dos seis compostos de interesse (BTEX) variando o tipo de solvente utilizado, o tempo e a velocidade de extração; as duas últimas etapas consistiram, respectivamente, no tratamento estatístico dos dados obtidos e na validação da metodologia, segundo os seguintes parâmetros: seletividade, faixa de trabalho, linearidade, limite de detecção, limite de quantificação e de recuperação.

\subsection{Otimização das condições da análise cromatográfica}

Foram testadas duas colunas cromatográficas com polaridade de fases diferentes, coluna Supelcowax $^{\mathrm{TM}} 10$ e coluna HP-5. Para ambas as colunas foram utilizadas as seguintes condições cromatográficas para a análise de Benzeno, Tolueno, Etilbenzeno e Xilenos:

Equipamento HP 6890, gás de arraste He com fluxo de $1,8 \mathrm{~mL} \mathrm{~min}^{-1}$; rampas de temperatura - inicial: $40{ }^{\circ} \mathrm{C}$ por 1 minuto, rampa $1: 10^{\circ} \mathrm{C} \min ^{-1}$ até $90{ }^{\circ} \mathrm{C}$, rampa $2: 4{ }^{\circ} \mathrm{C} \min ^{-1}$ até $120{ }^{\circ} \mathrm{C}$, rampa 3: $20{ }^{\circ} \mathrm{C} \min ^{-1}$ até $200{ }^{\circ} \mathrm{C}$ por 1 minuto; temperatura do injetor $240{ }^{\circ} \mathrm{C}$; modo de injeção: com divisão de fluxo (Split) 1:10; detector de ionização por chama: temperatura do detector: $240{ }^{\circ} \mathrm{C}$; injetor automático HP 7683; volume de injeção: $1 \mu \mathrm{L}$.

A concentração de trabalho utilizada foi de $10 \mathrm{mg} \mathrm{L}^{-1}$ e os tempos de retenção foram obtidos injetando-se os compostos BTEX, na forma de soluções individuais, na concentração de $1000 \mathrm{mg} \mathrm{L}^{-1}$.

\subsection{Otimização das condições da extração}

A extração líquido-líquido foi utilizada como método de extração. Foram testados dois tipos de solventes em diferentes tempos de extração e velocidades de agitação. Utilizou-se $25,00 \mathrm{~mL}$ de água fortificada com os compostos de interesse na concentração de $200 \mu \mathrm{g} \mathrm{L}^{-1} \mathrm{e}$ $250 \mu \mathrm{L}$ do solvente de extração, com fator de concentração de 100 vezes. As condições testadas na microextração líquido-líquido para a análise de Benzeno, Tolueno, Etilbenzeno e Xilenos podem ser observadas na Tabela 2.

Tabela 2. Condições da microextração tipo líquido-líquido na análise de Benzeno, Tolueno, Etilbenzeno e Xilenos em água fortificada.

\begin{tabular}{|c|c|c|c|}
\hline Solvente & 1-octanol & 1-heptanol & Isoctano \\
\hline Condições & $\begin{array}{l}\text { Tempo: } 35 \text { minutos } \\
\text { Velocidade de agitação: } \\
700 \text { rpm }\end{array}$ & $\begin{array}{l}\text { Tempo: } 35 \text { minutos } \\
\text { Velocidade de agitação: } \\
700 \text { rpm }\end{array}$ & $\begin{array}{l}\quad 6 \text { tempos: } \\
\text { 15, 20, 25, 30, 35, } 40 \text { minutos } \\
\text { 4 velocidades de agitação: } \\
650,700,750,800 \mathrm{rpm}\end{array}$ \\
\hline
\end{tabular}

\section{RESULTADOS E DISCUSSÃO}

\subsection{Comparação entre as colunas cromatográficas}

A coluna HP-5, apesar de ser amplamente utilizada na análise de BTEX, não separou os isômeros meta e p-Xileno. Trabalhos mais recentes como o apresentado por Kelly e Harder (2003) mostram que a coluna Supelcowax ${ }^{\mathrm{TM}} 10$, de fase polar, separa os isômeros do Xileno e ainda possui a vantagem de ter um menor tempo de análise em comparação com o tempo obtido pela coluna apolar HP-5.

Em uma separação cromatográfica há duas propriedades principais responsáveis pelos tipos de interação: a pressão de vapor do soluto (na temperatura da coluna) e a interação físico-química do soluto com a fase estacionária. Quando a fase da coluna cromatográfica é 
apolar, a separação ocorre mais pelo fenômeno físico da pressão de vapor dos compostos de interesse do que propriamente pelo princípio de interação físico-química entre a fase estacionária da coluna cromatográfica e os compostos. Quando a coluna é polar há a presença de sítios ativos na fase estacionária o que potencializa o princípio de adsorção e, consequentemente, a interação dos analitos com a fase estacionária polar. Isto ocorre porque a composição química da fase estacionária afeta o processo de separação devido aos diferentes tipos de interação: dipolo-dipolo, dipolo-dipolo induzido, entre outros (Silva et al., 2005).

No caso dos BTEX, como as pressões de vapor para os Xilenos é a mesma para os isômeros para e meta, eles se separam melhor na coluna polar. Como o isômero orto tem pressão de vapor diferente da pressão de vapor dos isômeros meta e para, ele tem boa separação nas duas colunas.

A Tabela 3 mostra os tempos de retenção dos compostos BTEX na coluna HP-5 e na Suplecowax ${ }^{\mathrm{TM}} 10$, respectivamente.

Tabela 3. Tempos de retenção $\left(t_{r}\right)$ dos compostos BTEX na coluna HP-5 e na coluna Supelcowax ${ }^{\mathrm{TM}} 10$.

\begin{tabular}{l|cc}
\hline Composto & $\begin{array}{c}\text { HP-5 } \\
\mathbf{t}_{\mathbf{R}}(\mathbf{m i n u t o s})\end{array}$ & $\begin{array}{c}\text { Supelcowax } \\
\mathbf{t}_{\mathbf{R}}(\mathbf{m i n u t o s})\end{array}$ \\
\hline Benzeno & - & 3,75 \\
Tolueno & 3,43 & 4,86 \\
Etilbenzeno & 4,56 & 6,02 \\
o-Xileno & 4,96 & 6,96 \\
m-Xileno & 4,63 & 6,25 \\
p-Xileno & 4,64 & 6,15 \\
\hline
\end{tabular}

A Figura 1 mostra um cromatograma dos compostos BTEX analisados na coluna Supelcowax ${ }^{\mathrm{TM}} 10$, com seus respectivos tempo de retenção mostrando a separação adequada dos seis analitos de interesse.

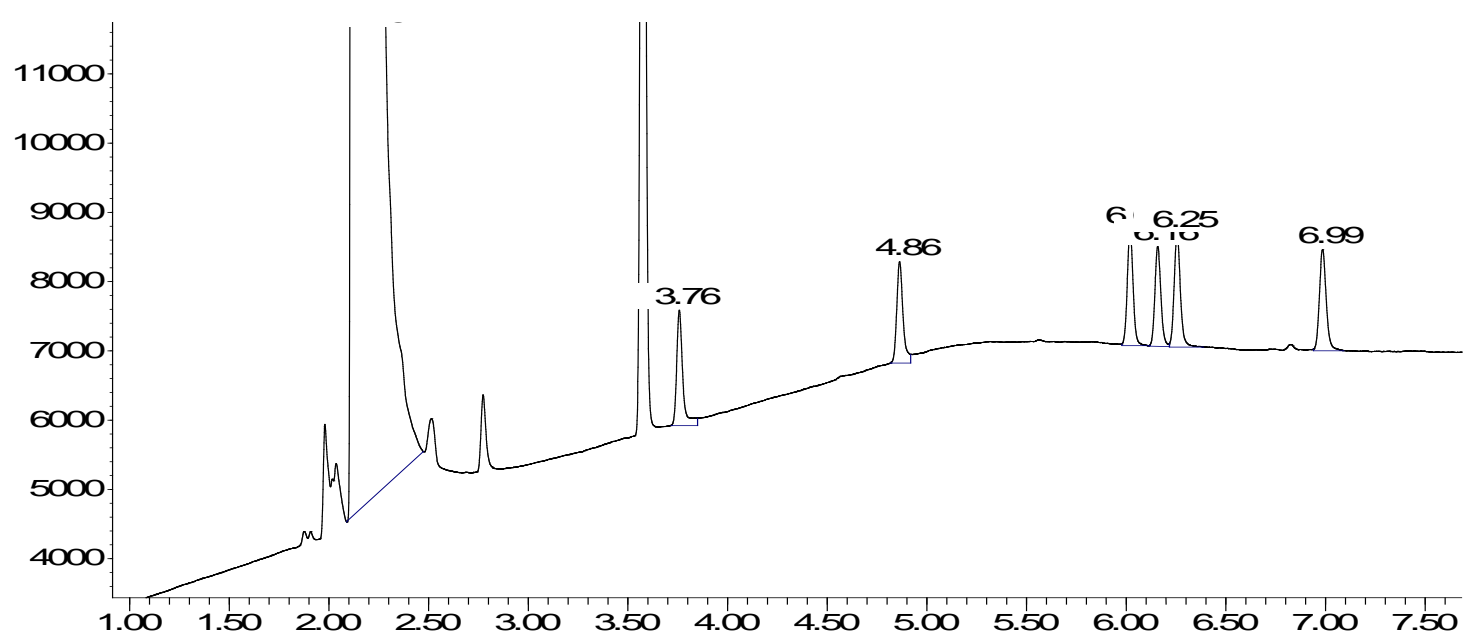

Figura 1. Cromatograma dos compostos Benzeno $(3,76)$, Tolueno $(4,86)$, Etilbenzeno $(6,02)$, o-Xileno $(6,99)$, m-Xileno $(6,25)$ e p-Xileno $(6,16)$ na coluna Supelcowax ${ }^{\text {TM }} 10$. 


\subsection{Metodologias de extração}

\subsubsection{Microextração Líquido-Líquido}

O solvente 1-octanol apresentou impurezas impossibilitando a sua utilização como solvente de extração. O solvente 1-heptanol, além de possuir uma impureza com tempo de retenção muito próximo ao analito o-Xileno, também mostrou que, por efeito de polaridade, os compostos que eluem da coluna mais próximos à eluição do solvente apresentam picos com resolução inadequada pela presença de "barriga" no cromatograma. O solvente isooctano foi o que apresentou melhores resultados de extração, com um cromatograma com picos bem resolvidos e bem definidos. Deste modo, este foi o solvente de extração escolhido.

Após a escolha do solvente de extração foram realizados testes para a escolha do melhor tempo de extração. O resultado obtido pode ser observado na Figura 2. Houve um aumento da eficiência de extração quando aumentado o tempo de extração em até 35 minutos. A partir deste tempo houve um decréscimo na eficiência de extração. Isso ocorreu por evaporação do solvente orgânico ou pela sua dissolução na fase aquosa (Sarafraz-Yazdi et al., 2009).

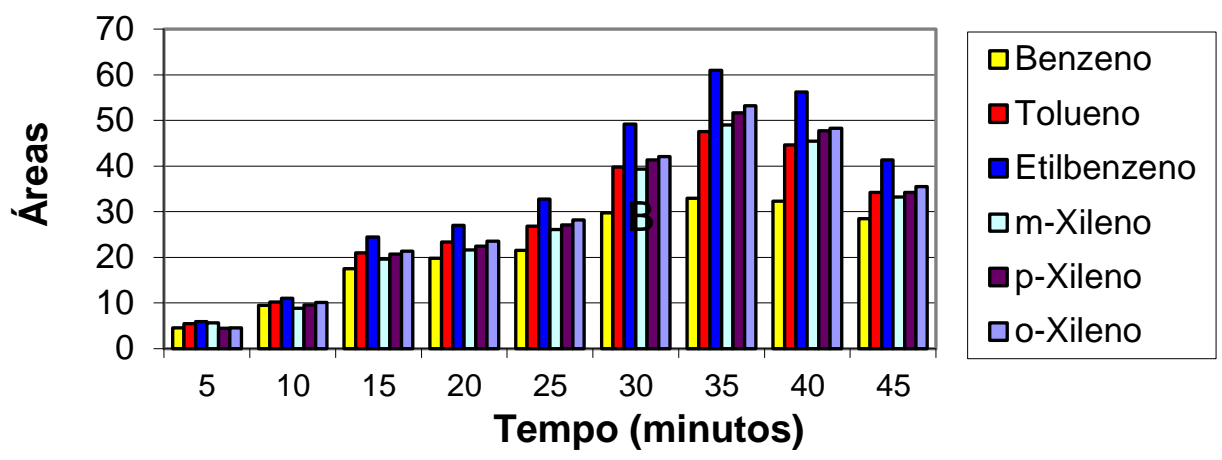

Figura 2. Área e Tempo de Extração para água fortificada com mistura dos analitos, concentração de $200 \mu \mathrm{g} \mathrm{L}{ }^{-1}$, com velocidade de extração constante e fixa de $700 \mathrm{rpm}$.

Após a escolha do solvente e do melhor tempo de extração foram realizados testes para a escolha da melhor velocidade de extração. O resultado obtido pode ser observado na Figura 3. Houve um aumento da eficiência de extração quando aumentada a velocidade de agitação até um valor de $700 \mathrm{rpm}$. A partir desse valor houve um decréscimo na eficiência de extração devido a maior transferência dos analitos voláteis da fase líquida para fase gasosa.

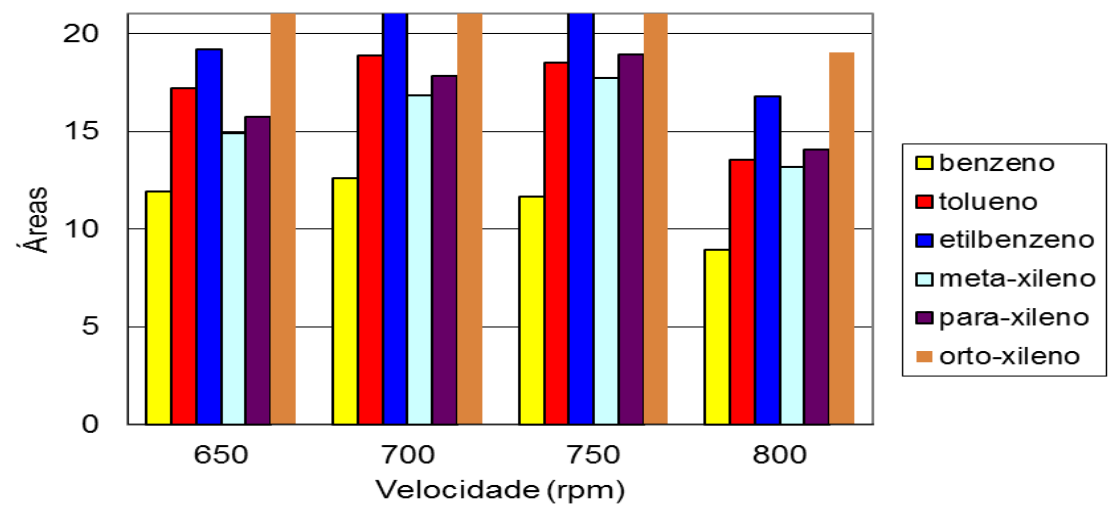

Figura 3. Área e velocidade de agitação para água Tipo1 fortificada com mistura dos analitos, concentração de $200 \mu \mathrm{g} \mathrm{L}^{-1}$, com tempo de extração de 35 minutos. 


\subsection{Validação das metodologias analíticas}

\subsubsection{Seletividade:}

A retenção relativa foi usada para identificação de substâncias e pode ser calculada para quaisquer picos no cromatograma, livre da restrição de serem adjacentes. Os valores de tempo de retenção $\left(t_{R}\right)$, tempo de retenção relativo $\left(t^{\prime}{ }_{R}\right)$ e fator de separação $(\alpha)$ dos seis analitos de interesse, podem ser observados na Tabela 4.

Tabela 4. Coeficientes de seletividade para os compostos BTEX(s).

\begin{tabular}{l|ccc}
\hline Composto & $\mathbf{t}_{\mathbf{R}}$ & $\mathbf{t}_{\mathbf{R}}$ & $\boldsymbol{\alpha}$ \\
\hline Benzeno & 3,75 & 1,56 & 1,7 \\
Tolueno & 4,86 & 2,67 & 1,4 \\
Etilbenzeno & 6,02 & 3,83 & 1,2 \\
o-Xileno & 6,96 & 4,77 & 0,9 \\
m-Xileno & 6,25 & 4,06 & 1,0 \\
p-Xileno & 6,15 & 3,96 & 1,0 \\
\hline
\end{tabular}

\subsubsection{Faixa de trabalho:}

A faixa de trabalho de 10 a $50 \mu \mathrm{g} \mathrm{mL}^{-1}$ demonstrou-se adequada para construção das curvas analíticas, segundo os critérios de aceitabilidade da linearidade. Essa faixa de trabalho atende os limites de concentração máxima permitidos para cinco dos seis analitos de interesse.

\subsubsection{Linearidade:}

A utilização do método de regressão linear exige que algumas premissas sejam verdadeiras de modo a garantir que essa seja a melhor escolha do modelo matemático a ser utilizado para avaliação dos dados obtidos. Neste trabalho, as seguintes premissas foram avaliadas: valores extremos, normalidade, autocorrelação de resíduos, desvio de linearidade (falta de ajuste) e homogeneidade de variâncias.

Para detecção dos valores discrepantes foram utilizados os testes de Jack-Knife para um nível de significância estatística de 5\%. Para o Benzeno, dois valores extremos foram detectados e, como estavam causando desvios de linearidade foram rejeitados. Para os outros cinco compostos, um valor extremo foi detectado para cada um dos mesmos, com significância de $5 \%$ e n=4, como não causavam desvios de linearidade não foram rejeitados.

Para avaliar a normalidade dos resíduos foi utilizado o teste de Ryan-Joiner, que testa se os dados provêm ou não de uma distribuição normal. Após a retirada dos dois valores extremos para a construção da curva analítica do Benzeno, todos os seis compostos passaram nos testes de normalidade dos resíduos realizados com o teste de Ryan-Joiner, com significância de $5 \%$.

Para verificação da existência de autocorrelação dos resíduos foi utilizado o teste mais conhecido e empregado, o Durbin-Watson. Pelo teste verificou-se que não há autocorrelação dos resíduos em nenhum dos seis analitos de interesse, demonstrando assim, que o modelo de regressão linear pode ser utilizado.

Como última premissa verificada para a utilização do método dos mínimos quadrados foi verificada a homogeneicidade dos sistemas para cada um dos seis analitos de interesse, pelo teste de Levene. Para os seis analitos de interesse os valores de p encontrados foram maiores que 0,05 indicando que há homogeneicidade nos sistemas. 
Deste modo, as curvas analíticas dos seis analitos em questão foram construídas pelo método dos mínimos quadrados ordinários.

\subsubsection{Limite de Detecção:}

Foram calculados empregando-se o método baseado em parâmetros da curva analítica segundo Aragão et al. (2009). Neste método, o Limite de Detecção pode ser calculado a partir da Equação 1 e os valores obtidos estão apresentados a seguir.

$$
\mathrm{LD}=(3,3 \times \mathrm{s}) / \mathrm{a}
$$

em que:

S - é a estimativa do desvio padrão da resposta (que pode ser a estimativa do desvio padrão do branco, da equação da linha de regressão ou do coeficiente linear da equação); $\mathrm{e}$

a - é o coeficiente angular da curva analítica.

Foram obtidos os seguintes valores de Limite de Detecção (LD) para os analitos de interesse em $\mu \mathrm{g} \mathrm{mL}^{-1}$ : Benzeno, 2,74; Tolueno, 0,85; Etilbenzeno, 0,90; o-Xileno, 0,96; m-Xileno, 0,90 e p-Xileno, 0,88.

\subsubsection{Limite de Quantificação:}

Foram calculados empregando-se o método baseado em parâmetros da curva analítica segundo Aragão et al. (2009). Neste método, o Limite de Quantificação pode ser calculado a partir da Equação 2 e os valores obtidos estão a seguir:

$$
\mathrm{LQ}=(10 \times \mathrm{s}) / \mathrm{a}
$$

em que:

S - é a estimativa do desvio padrão da resposta (que pode ser a estimativa do desvio padrão do branco, da equação da linha de regressão ou do coeficiente linear da equação);e

a - é o coeficiente angular da curva analítica.

Foram obtidos os seguintes valores de Limite de Quantificação (LQ) para os analitos de interesse em $\mu \mathrm{g} \mathrm{mL}^{-1}$ : Benzeno, 8,29; Tolueno, 2,57; Etilbenzeno, 2,74; o-Xileno, 2,91; m-Xileno, 2,72 e p-Xileno, 2,66;

\subsubsection{Construção das Curvas Analíticas:}

A curva de quantificação foi elaborada utilizando o método de curva de extração construída com a extração de água Tipo 1 fortificada com os analitos de interesse nas concentrações de 0,$10 ; 0,20 ; 0,30 ; 0,40$ e $0,50 \mu \mathrm{g} . \mathrm{L}^{-1}$. O objetivo era de obter as seguintes concentrações finais nos extratos após a concentração de 100 vezes: $10 ; 20 ; 30 ; 40$ e $50 \mu \mathrm{g} . \mathrm{L}^{-}{ }^{1}$.

\subsubsection{Recuperação:}

Para o estudo de recuperação foram fortificadas amostras de água Tipo1, com solução contendo todos os analitos de interesse, de modo que a solução final, após a extração, tivesse os analitos nas concentrações de $10 ; 30$ e $50 \mu \mathrm{g} \mathrm{L}^{-1}$. Quando a recuperação é obtida a partir de fortificações de matriz branca, o fator de recuperação $\left(f_{\text {rec }}\right)$ é calculado pela Equação 3 (Brasil, 2011a): 


$$
f_{\text {rec }}=\left[\left(\mathrm{C}_{\mathrm{f}}-\mathrm{C}_{\mathrm{nf}}\right) / \mathrm{C}_{\mathrm{ad}}\right] \times 100
$$

Os valores de percentual de Recuperação para os analitos de interesse podem ser observados na Tabela 5:

Tabela 5. Valores de Recuperação cientes para os compostos BTEX(s).

\begin{tabular}{l|ccc}
\hline Composto & $\begin{array}{c}\text { Recuperação(\%) } \\
\mathbf{1 0} \boldsymbol{\mu g . L}^{-\mathbf{1}}\end{array}$ & $\begin{array}{c}\text { Recuperação (\%) } \\
\mathbf{3 0} \boldsymbol{\mu g . L}^{-\mathbf{1}}\end{array}$ & $\begin{array}{c}\text { Recuperação (\%) } \\
\mathbf{5 0} \boldsymbol{\mu g . L}^{-\mathbf{1}}\end{array}$ \\
\hline Benzeno & 78,3 & 90,2 & 97,2 \\
Tolueno & 92,5 & 97,9 & 100,1 \\
Etilbenzeno & 90,5 & 98,9 & 99,3 \\
o-Xileno & 90,0 & 99,2 & 99,5 \\
m-Xileno & 90,8 & 99,5 & 99,7 \\
p-Xileno & 90,7 & 100,1 & 99,8 \\
\hline
\end{tabular}

Neste trabalho foi testado o efeito da adição de sal na eficiência da extração em amostras de água fortificada. Os resultados mostraram-se em concordância com Zanjani et al. (2007), indicando que a eficiência de extração dos compostos BTEX não se alterou pela adição de $\mathrm{NaCl}$ à amostra fortificada; por isso, as extrações foram realizadas na ausência do sal.

\section{CONCLUSÃO}

Neste trabalho foi desenvolvida e implementada uma nova metodologia para extração e determinação de resíduos de Benzeno, Tolueno, Etilbenzeno e Xilenos, em água de abastecimento público. Os limites de quantificação obtidos atendem aos valores máximos estabelecidos pela Norma de Potabilidade, do Ministério da Saúde (Portaria No 2914/2011), para cinco dos seis compostos estudados, ficando acima apenas para o benzeno.

A necessidade de um pequeno volume de amostra e de reagentes, e a não inclusão da etapa de evaporação do extrato permite um procedimento rápido, fácil e relativamente pouco oneroso, o que torna a metodologia estudada adequada às análises de rotina para controle da qualidade da água para consumo humano.

Tendo em vista que os analitos estudados são voláteis, o uso de uma técnica de extração que permite a concentração da amostra no próprio procedimento diminui as perdas durante o processo. A técnica de microextração líquido-líquido também reduz o limite de quantificação, pois, é possível pré concentrar as amostras em 100 vezes durante o processo de extração.

Mediante o uso de uma coluna com fase de separação polar (Supelcowax ${ }^{\mathrm{TM}} 10$ ), todos os compostos foram separados com resolução de separação adequada e curto tempo de análise, fato pouco reportado na literatura.

A coluna Supelcowax ${ }^{\mathrm{TM}} 10$ separa com resolução adequada todos os isômeros do Xileno, pois, estes isômeros interagem de forma diferente com a fase polar da coluna. A separação dos isômeros é importante porque existem modelos toxicocinéticos de exposição por inalação de Xileno em humanos que sugerem que há diferenças significativas entre a toxicidade, meia-vida, depuração e metabolismo entre as formas isoméricas dos Xilenos. Outros estudos indicam diferentes ordens de toxicidade relativa para os isômeros e as diferenças nos níveis de vigor, mesmo sendo pequenas. Assim, uma metodologia capaz de quantificar a separação dos isômeros posicionais do xileno pode ser interessante para diferenciar e entender melhor a exposição humana e ambiental ao m-Xileno e do p-Xileno. 
Os dados obtidos foram tratados estatisticamente com resultados satisfatórios em todos os testes realizados.

Em relação à validação da metodologia, todos os parâmetros de desempenho do método estudado apresentaram-se em concordância com o estabelecido pelos órgãos reguladores, INMETRO e ANVISA, demonstrando que a metodologia é adequada ao objetivo proposto.

\section{REFERÊNCIAS}

AGÊNCIA NACIONAL DE VIGILÂNCIA SANITÁRIA (ANVISA). Guia para qualidade em química analítica - uma assistência à habilitação. Brasília, DF, 2005.

ARAGÃO, N. M.; VElOSO, M. C. C.; ANDRADE, J. B. Validação de métodos cromatográficos de análise - um experimento de fácil aplicação utilizando cromatografia líquida de alta eficiência (CLAE) e os princípios da "química verde" na determinação de metilxantinas em bebidas. Química Nova, v. 32, n. 9, p. 2476-2481, 2009. http://dx.doi.org/10.1590/S0100-40422009000900043

ASSOCIAÇÃO BRASILEIRA DE NORMAS TÉCNICAS. NBR ISO/IEC 17.025. Requisitos gerais para competência de laboratórios de ensaio e calibração. Rio de Janeiro, 2005.

AUSTRALIA. National Health and Medical Research Council. Australian drinking water guidelines (ADWG). National water quality management strategy. Canberra, 2004. $511 \mathrm{p}$.

BRASIL. Ministério da Agricultura, Pecuária e Abastecimento. Guia de validação e controle de qualidade analítica (CGAL/SDDA). Fármacos em Produtos para Alimentação Animal e Medicamentos Veterinários. Brasília, 2011.72p.

BRASIL. Ministério da Saúde. Portaria N 2914, de 12 de dezembro de 2011. Estabelece os procedimentos e responsabilidades relativos ao controle e vigilância da qualidade da água para consumo humano e seu padrão de potabilidade, e dá outras providências. Diário Oficial [da] União, Poder Executivo, de 14 de dezembro de 2011.

BRASIL. Ministério do Meio Ambiente. Conselho Nacional do Meio Ambiente (CONAMA). Resolução No. 357, de 17 de março de 2005. Dispõe sobre a classificação dos corpos de água e diretrizes ambientais para o seu enquadramento, bem como estabelece as condições e padrões de lançamento de efluentes, e dá outras providências. Diário Oficial [da] União, de 18 de março de 2005.

CANADA. Health Canada. Guidelines for canadian drinking water quality (GCDWQ). Supporting Document. $6^{\text {th }}$ Ed. Ontario, 1996. 90 p.

CORSEUIL, H. X.; MARINS, M. D. M. Contaminação de águas subterrâneas por derramamentos de gasolina: O problema é grave? Revista Engenharia Sanitária e Ambiental, v. 2, n. 2, p. 50-54, 1997.

EURACHEM. The fitness for purpose of analytical methods, a laboratory guide to method validation and related topics. Teddington: LGC, 1998. 61p.

EUROPEAN COMMUNITIES. Drectiva 98/83/EC. On the quality of water intended for human consumption. Official Journal of the European Communities, 5 dez. 1998. Disponível em: <http://ec.europa.eu> Acesso em: 26 fe.v 2014. 
GOBATO, E. A. A. F.; LANÇAS, F. M. Comparação entre injeção na coluna (on-column) e headspace dinâmico na determinação de Benzeno, Tolueno e Xilenos (BTX) em amostras de água. Química Nova, v. 24, n. 2, p. 176-179, 2001. http://dx.doi.org/10.1590/S0100-40422001000200004

GREEN, J. M. A practical guide to analytical method validation. Analytical Chemistry, v. 68, p. 305A-309A, 1996. http://dx.doi.org/10.1021/ac961912f

INSTITUTO NACIONAL DE METROLOGIA (INMETRO). DOQ-CGCRE-008 Orientações sobre validação de métodos de ensaios químicos. Brasília, 2003. 35 p.

KELLY, K.; HARDER, B. BTEX(s): Complete resolution in under 6.5 minutes. Environmental. The applications book, Torrance, Phenomenex p 1-2, April 2003.

LOURENÇO, M. Realização periódica de análises químicas de BTEX e PAH, em amostras de água e solo, nos postos de combustíveis. 2005. Disponível em: <http://www.portaldepostos.com.br/paginas/gest.meioambiente.materia9.html>. Acesso em: 26 fev. 2014.

SARAFRAZ-YAZDI, A.; AMIRI, A. H.; ES'HAGHI, Z. Separation and determination of Benzene, Toluene, Ethybenzene and o-Xylene compounds in water using directly suspended droplet microextraction coupled with chromatography-flame ionization detector. Talanta, v. 78, p. 936-941, 2009. http://dx.doi.org/10.1016/j. talanta.2008.12.069

SILVA, D. D.; INNOCENTINI, A. P.; CHIERICE, G. O.; GALHIANE, M. S.; SANTOS, L. S. Avaliação da seletividade de uma nova coluna capilar em cromatografia gasosa teste de Grob e índice de retenção. Revista Analytica, n. 17, p. 40-47, 2005.

SILVA, R. L. B. Contaminação de poços rasos no bairro Brisamar, Itaguai, RJ, por derramamento de gasolina: concentração de BTEX(s) e avaliação da qualidade da água consumida pela população. 2002. Tese (Doutorado) - Escola Nacional de Saúde Pública Sergio Arouca, Fundação Oswaldo Cruz, Rio de Janeiro, 2002.

SILVA, R. L. B.; BARRA, C. M.; MONTEIRO, T. C. N.; BRILHANTE, O. M. Estudo da contaminação de poços rasos por combustíveis orgânicos e possíveis consequências para a saúde pública no Município de Itaguaí, Rio de Janeiro, Brasil. Cadernos de Saúde Pública, v. 18, n. 6, p. 1599-1607, nov./dez., 2002. http://dx.doi.org/10.1590/ S0102-311X2002000600014

UNITED STATES. Environmental Protection Agency (USEPA). List of Contaminants \& their Maximum Contaminant Level (MCLs) in drinking water. Washington (DC), 2004.

WORLD HEALTH ORGANIZATION (WHO). Air quality guidelines. Second Edition, Chapter 5. 2. Benzene. Copenhagen, Denmark, 2000a.

WORLD HEALTH ORGANIZATION (WHO). Air quality guidelines. Second Edition, Chapter 5. 14. Toluene. Copenhagen, Denmark, $2000 \mathrm{~b}$.

WORLD HEALTH ORGANIZATION (WHO). Guidelines for drinking-water quality. Ethylbenzene in Drinking-Water. Second Edition, Vol. 2. Health Criteria and other Supporting Information. Geneva, 1996a. 
WORLD HEALTH ORGANIZATION (WHO). Guidelines for drinking-water quality. Benzene in Drinking-Water. Second Edition, Vol. 2. Health Criteria and other Supporting Information. Geneva, $1996 \mathrm{~b}$.

WORLD HEALTH ORGANIZATION (WHO). Guidelines for drinking-water quality. Toluene in Drinking-Water. Second Edition, Vol. 2. Health Criteria and other Supporting Information. Geneva, 2004.

ZANJANI, M. R. K.; YAMINI, Y.; SHARIATI, S.; JONSSON J. A. A new liquid-phase microextraction method based on solidification of floating organic drop. Analytica Chimica Acta, v. 585, p. 286-293, 2007. http://dx.doi.org/10.1016/j.aca.2006.12.049 